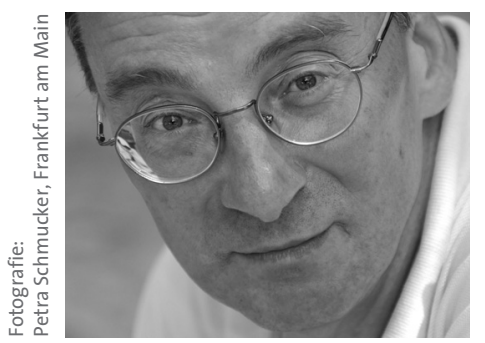

\title{
Input, Output, Outcome
}

Das Lexikon hilft wie so oft nicht viel weiter: »Wertschöpfung, Summe der in einem Unternehmen oder einer Volkswirtschaft während einer Periode geschaffenen wirtschaftli. Werte. (Brockhaus 2004) Alles hängt bei dieser Definition davon ab, wer welchen Produkten und Dienstleistungen einen wie hohen » Wert « beimisst. Ein Verkäufer mag das vielleicht anders sehen als ein Kunde, ein Geschäftsführer anders als ein Mitarbeiter, ein Politiker anders als ein Steuerzahler. Wie sich das sozialwirtschaftliche Verständnis von einer betriebswirtschaftlich geprägten Wertschöpfung unterscheidet, zeigt Wolf Rainer Wendt in seinem einleitenden Beitrag zu diesem Heft: Im Produktionsprozess eines sozialwirtschaftlichen Unternehmens sollte sich das Handeln auf eine Wertschöpfung » am Menschen « konzentrieren. Nur wenn Personen und Gesellschaft einen Nutzen aus sozialen Diensten und Einrichtungen ziehen, kann in diesem Sinne von »Wertschöpfung " gesprochen werden. Dass der in Geldbeträgen bezifferbare Output eines Betriebs am Jahresende größer ist als der entsprechende Input, besagt noch nichts über die erreichte Lebensqualität für die Menschen, für den die Organisation da sein will. Erkennbar und zu beurteilen sei dieser Ertrag, so Wendt, am Outcome (also an den Wirkungen) von zielführenden Prozessen, nicht am Output (also den bloßen Produkten und Dienstleistungen), und, was die innerbetriebliche Wertschöpfung betreffe, am Throughput (also den innerbetrieblichen Strukturen und Prozessen) eines schrittweisen Faktoreinsatzes, mag er auch - und damit die betriebliche Leistungserbringung - mehr oder weniger effektiv und effizient erfolgen.

Die Vorbereitungen für den nächsten Kongress der Sozialwirtschaft laufen. Termin und Ort stehen bereits fest: Die siebte Veranstaltung dieses Branchentreffen findet am 26. und 27. Mai 2011 in Magdeburg. Als Rahmenthema zeichnet sich ab, wie sich die Sozialwirtschaft nach der Finanz- und Wirtschaftskrise neu positionieren kann. Methodisch soll bei diesem Treffen von rund 400 Verantwortlichen besonderen Wert gelegt werden auf die Vernetzung der Veranstalter, der Referenten und der Teilnehmenden. Zudem soll ein besonderes Augenmerk gelegt werden auf praktische Beispiele aus gewerblicher Wirtschaft und freier Wohlfahrtspflege. Eine Website informiert kontinuierlich über den Stand der Vorbereitungen: http://www.kongressder-sozialwirtschaft.de. 